\title{
Short report: interim safety results for a phase II trial measuring the integration of stereotactic ablative radiotherapy (SABR) plus surgery for early stage non-small cell lung cancer (MISSILE-NSCLC)
}

David A. Palma ${ }^{1,2^{*}}$, Timothy K. Nguyen ${ }^{1}$, Keith Kwan ${ }^{3}$, Stewart Gaede ${ }^{2,4,5}$, Mark Landis ${ }^{6}$, Richard Malthaner ${ }^{7}$, Dalilah Fortin ${ }^{7}$, Alexander V. Louie ${ }^{1,2}$, Eric Frechette ${ }^{7}$, George B. Rodrigues ${ }^{1,2}$, Brian Yaremko ${ }^{1,2}$, Edward Yu $u^{1,2}$, A. Rashid Dar ${ }^{1,2}$, Ting-Yim Lee ${ }^{4,8}$, Al Gratton ${ }^{1}$, Andrew Warner ${ }^{1}$, Aaron Ward ${ }^{2,4}$ and Richard Inculet ${ }^{7}$

\begin{abstract}
A phase II trial was launched to evaluate if neoadjuvant stereotactic ablative radiotherapy (SABR) before surgery improves oncologic outcomes in patients with stage I non-small cell lung cancer (NSCLC). We report a mandated interim safety analysis for the first 10 patients who completed protocol treatment. Operable patients with biopsy-proven T1-2 NO NSCLC were eligible. SABR was delivered using a risk-adapted fractionation (54Gy/3 fractions, 55/5 or 60/8). Surgical resection was planned 10 weeks later at a high-volume center (>200 lung cancer resections annually). Patients were imaged with dynamic positron emission tomography-computed tomography scans using ${ }^{18}$ F-fludeoxyglucose $\left({ }^{18} \mathrm{~F}\right.$-FDG-PET CT) and dynamic contrast-enhanced CT before SABR and again before surgery. Toxicity was recorded using CTCAE version 4.0. Twelve patients were enrolled between 09/2014 and 09/2015. Two did not undergo surgery, due to patient or surgeon preference; neither patient has developed toxicity or recurrence. For the 10 patients completing both treatments, median age was 70 (range: 54-76), 60\% had $\mathrm{T} 1$ disease, and $60 \%$ had adenocarcinoma. Median $\mathrm{FEV}_{1}$ was $73 \%$ predicted (range: $54-87 \%$ ). Median time to surgery post-SABR was 10.1 weeks (range: 9.3-15.6 weeks). Surgery consisted of lobectomy $(n=8)$ or wedge resection $(n=2)$. Median follow-up post-SABR was 6.3 months. After combined treatment, the rate of acute grade 3-4 toxicity was 10\%. There was no post-operative mortality at 90 days. The small sample size included herein precludes any definitive conclusions regarding overall toxicity rates until larger datasets are available. However, these data may inform others who are designing or conducting similar trials.
\end{abstract}

Trial registration: NCT02136355. Registered 8 May 2014.

Keywords: Non-small cell, Lung cancer, Stereotactic ablative radiotherapy, Stereotactic body radiotherapy, Surgery

\section{Introduction}

Stereotactic ablative radiotherapy (SABR, also called stereotactic body radiation therapy or SBRT) provides a promising therapeutic option for stage I non-small cell lung cancer (NSCLC) [1]. Many studies report 3-year local control rates of approximately $90 \%$, based on post-

\footnotetext{
* Correspondence: David.Palma@lhsc.on.ca

'Department of Radiation Oncology, London Health Sciences Centre, London, Canada

2Department of Oncology, Western University, London, Canada

Full list of author information is available at the end of the article
}

treatment imaging [2]. However, the true pathologic complete response rate $(\mathrm{pCR})$ is unknown. In most patients, local radiation-induced lung injury (RILI) is evident on CT after SABR and can impair the detection of local recurrences [3].

In operable patients undergoing surgery, neoadjuvant SABR could theoretically provide an oncologic benefit by decreasing the rate of positive margins, sterilizing the tumor to avoid the seeding of circulating tumor cells during surgery [4], or in some cases through an abscopal effect [5]. The MISSILE-NSCLC study was launched to 
evaluate the oncologic outcomes and toxicity after an $a$ priori combined treatment approach of SABR followed by surgical resection, and to determine the $\mathrm{pCR}$ rate after SABR.

To our knowledge, the toxicity of this combined treatment approach has not been previously reported. Herein, we present the findings of an interim safety analysis.

\section{Methods}

\section{Study design and patient selection}

Institutional Research Ethics Board approval was obtained prior to study initiation, and the study was centrally registered (NCT02136355). Eligible patients had histologically-confirmed $\mathrm{T} 1$ or T2a $(\leq 5 \mathrm{~cm})$ NSCLC, with no evidence of nodal or distant metastases. Additional requirements included age $\geq 18$, informed consent, Eastern Cooperative Oncology Group (ECOG) performance status $0-2$, life expectancy $>6$ months and a predicted post-operative forced expiratory volume in 1 second $\left(\mathrm{FEV}_{1}\right)$ of $\geq 30 \%$. Ineligibility criteria included patients with contraindication to either treatment, previous lung cancer within the past 5 years, previous thoracic radiation or a contrast allergy.

\section{Interventions}

SABR was delivered according to a risk-adapted proto$\mathrm{col}$, with the dose and fractionation dependent on the size and location of the tumor (54 Gy / 3 fractions, 55 Gy/5 fractions or 60 Gy/8 fractions). Treatment was delivered every second day regardless of the dosefractionation regimen [6, 7]. A 4-D CT simulation scan was acquired for all patients. Respiratory gating was considered in cases where motion was $>7 \mathrm{~mm}$ in any direction.

The gross tumor volume (GTV) was defined as the visible tumor on $\mathrm{CT}$ imaging $\pm \mathrm{PET}$, and an internal GTV encompassed the GTV from all phases of respiration. No additional margin was included for microscopic spread of disease. A planning target volume (PTV) margin of $5 \mathrm{~mm}$ was used. The prescription point was approximately the $80 \%$ isodose line surrounding the PTV, with the requirement that $95 \%$ of the PTV was covered by $100 \%$ of the prescription dose.

Surgical resection was planned to occur at $10 \pm 2$ weeks following SABR and consisted of a lobectomy or sublobar resection by either an open or video-assisted thoracoscopic approach. All patients received sampling of high risk hilar and mediastinal lymph nodes at the time of resection. All procedures were done at a high-volume tertiary surgical centre, with a case volume of $>200$ lung resections annually.

Patients with pathologic node-positive disease (N1, $\mathrm{N} 2$, or N3) were referred for adjuvant chemotherapy.
For patients with N2 or N3 disease, adjuvant radiotherapy to the mediastinum was to be considered as long as there was minimal overlap with the SABR dose distribution.

The trial protocol mandated that after 10 patients have been accrued and completed surgery, a safety analysis to review treatment toxicity would be undertaken separately by the study team and the data safety monitoring committee.

\section{Results}

Twelve patients were enrolled between September 2014 and September 2015. Two did not undergo surgery following SABR due to concerns regarding medical operability: one continued to smoke more than two packs per day; the other had unrelated upper gastrointestinal dysfunction and possible gastroparesis resulting in poor functional status. Neither patient has developed toxicity or recurrence.

\section{Patient \& treatment characteristics}

For the 10 patients completing combined therapy, the median age was 70 (range: 54-76), 60\% had T1 disease, and $60 \%$ had adenocarcinoma. Median $\mathrm{FEV}_{1}$ was $73 \%$ predicted (range: $54-87 \%$ ). Median time to surgery postSABR was 10.1 weeks (range: 9.3-15.6 weeks). Most patients underwent a lobectomy $(n=8)$ post-SABR, with the remainder receiving a wedge resection $(n=2)$. Median follow-up post-SABR was 6.3 months.

\section{Outcomes}

A total of 24 toxicities were reported (Table 1). The most common toxicity was pain (grade 1-2), occurring in $90 \%$ of patients. Fatigue, pneumonia and pneumothorax each occurred in $20 \%$ of patients. There were 3 recorded grade 3-4 toxicities (pneumonia, atrial fibrillation and respiratory failure), all in the same patient, and all of which resolved. The highest grade of toxicity for each of the 10 patients is shown in Table 2. There were no deaths within 30 or 90 days of surgery.

\section{Discussion}

Neoadjuvant radiotherapy is part of standard treatment for certain cancers (e.g. rectal cancers, sarcomas), with the goal of reducing the risk of local recurrence or margin positivity. In stage I NSCLC, neoadjuvant ablative therapy has previously been explored using radiofrequency ablation (RFA) prior to surgical resection. However, the high prevalence of viable tumor cells after RFA (62\% of cases) discouraged its widespread adoption [8]. Although prior studies have evaluated salvage surgery in selected patients who developed local recurrence after SABR [9-12], the use of a combined approach upfront has not been assessed previously. 
Table 1 Type and grade of patient-reported toxicities

\begin{tabular}{|c|c|c|c|c|c|c|}
\hline \multirow[t]{2}{*}{ Toxicity } & \multicolumn{5}{|c|}{$\begin{array}{l}\text { Number of Patients Reporting Toxicity by Grade } \\
\text { ( } N=24 \text { total toxicities) }\end{array}$} & \multirow[t]{2}{*}{ Tota } \\
\hline & 1 & 2 & 3 & 4 & 5 & \\
\hline Pain & 2 & 7 & 0 & 0 & 0 & 9 \\
\hline Fatigue & 2 & 0 & 0 & 0 & 0 & 2 \\
\hline Pneumonia & 0 & 1 & 1 & 0 & 0 & 2 \\
\hline Pneumothorax & 0 & 2 & 0 & 0 & 0 & 2 \\
\hline Atrial Fibrillation (uncontrolled) & 0 & 0 & 0 & 1 & 0 & 1 \\
\hline Dyspnea & 1 & 0 & 0 & 0 & 0 & 1 \\
\hline Respiratory failure & 0 & 0 & 0 & 1 & 0 & 1 \\
\hline Post-Operative Bleeding & 1 & 0 & 0 & 0 & 0 & 1 \\
\hline Broncho-pleural Fistula & 0 & 1 & 0 & 0 & 0 & 1 \\
\hline Generalized Weakness & 1 & 0 & 0 & 0 & 0 & 1 \\
\hline Nausea & 0 & 1 & 0 & 0 & 0 & 1 \\
\hline Vomiting & 0 & 1 & 0 & 0 & 0 & 1 \\
\hline Anorexia & 1 & 0 & 0 & 0 & 0 & 1 \\
\hline
\end{tabular}

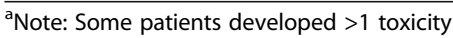

While the sample size in this interim report is small, the toxicity rates reported herein compare favorably with those in prospective studies evaluating surgery alone. In a pooled analysis looking at two randomized studies comparing SABR versus lobectomy, the rate of grade 35 toxicity after lobectomy was $48 \%$ [13]. Toxicity rates appear to be lower after sublobar resection, often reported as approximately 30\% [14]. However, comparisons across trials are subject to bias based on differences between study populations and treatments.

There is a strong relationship between hospital volume and surgical toxicity in NSCLC [15], and therefore the generalizability of these data to low-volume centers is unknown. In addition, the small sample size included herein precludes using the toxicity data to inform clinical practice until larger datasets are available. However, these data, compiled as a mandated interim safety report, are still informative. They sufficiently assure us it is safe to continue with combined treatment on the present clinical trial, which also provides reassurance to other groups who are designing or conducting similar trials. Furthermore, these promising early results

Table 2 Highest grade of reported toxicity for each of 10 patients

\begin{tabular}{ll}
\hline Grade & Highest Toxicity Grade for Each Patient $(n=10)$ \\
& N (\%) \\
\hline 1 & $1(10 \%)$ \\
3 & $7(70 \%)$ \\
4 & 0 \\
5 & $1(10 \%)$ \\
\hline
\end{tabular}

generate valuable academic discussion, which garner interest in an understudied area and primes interested parties to the forthcoming results of the present trial. If there is a demonstrable benefit to combined treatment, there will be a need for validation studies to confirm a potential shift in practice patterns. If results are negative, other groups may reexamine the concept with alternative methodology. In either case, subsequent steps will require time. By inciting discussion now we hope to expedite our ability to answer important clinical questions that ultimately inform the best possible care for our patients.

\section{Conclusion}

In conclusion, toxicity rates after combined SABR and surgical resection compare favorably with reported rates in prospective studies of surgical resection alone. While these findings are preliminary, and certainly inadequate to inform clinical practice, it provides an early assessment of the tolerability of combined treatment as we await mature data on $\mathrm{PCR}$ rates and oncologic outcomes.

Abbreviations

18 F-FDG: 18 F-fludeoxyglucose; CT: computed tomography; CTCAE: Common terminology criteria for adverse events; ECOG: Eastern Cooperative Oncology Group; FEV 1 : Forced expiratory volume in 1 second; GTV: gross tumor volume; NSCLC: non-small cell lung cancer;

pCR: pathologic complete response rate; PET: positron emission tomography; PTV: planning target volume; RFA: radiofrequency ablation; RILI: radiationinduced lung injury; SABR: stereotactic ablative radiotherapy;

SBRT: stereotactic body radiation therapy

Acknowledgements

DAP is supported by a Clinician-Scientist Grant from the Ontario Institute for Cancer Research. 


\section{Funding}

DAP is supported by a Clinician-Scientist Grant from the Ontario Institute for Cancer Research.

\section{Availability of data and materials}

The datasets generated during and/or analysed during the current study are not publicly available but may become available upon request with permission from corresponding author and host institution following sufficient maturation of data.

\section{Authors' contributions}

Introduction: DAP, TKN. Methodology/Study Design: DAP, KK, SG, ML, RM, GBR, BY, EY, TYL, AnW, AaW, RI. Data Collection: DAP, KK, SG, ML, RM, DF, AVL, EF, GBR, BY, EY, ARD, AG, AaW, Rl. Data Analysis: DAP, KK, ML, RM, TYL, AnW, AaW, Rl. Results: DAP, TKN, TYL, AnW, AaW, RI. Discussion: DAP, TKN, RM, AVL, GBR, BY, TYL, AaW, Rl. Final Proofing: All Authors. All authors read and approved the final manuscript.

\section{Competing interests}

The authors declare that they have no competing interests.

\section{Consent for publication}

Not applicable.

\section{Ethics approval and consent to participate}

Institutional Research Ethics Board approval was obtained from Lawson Health Research Institute prior to study initiation, and the study was centrally registered (NCT02136355).

\section{Author details}

'Department of Radiation Oncology, London Health Sciences Centre, London, Canada. ${ }^{2}$ Department of Oncology, Western University, London, Canada. ${ }^{3}$ Department of Pathology, Western University, London, Canada. ${ }^{4}$ Department of Medical Biophysics, Western University, London, Canada. ${ }^{5}$ Department of Physics and Engineering, London Health Sciences Centre, London, Canada. ${ }^{6}$ Department of Radiology, Western University, London, Canada. 'Department of Surgery, Division of Thoracic Surgery, Western University, London, Canada. ${ }^{8}$ Imaging Program, Lawson Health Research Institute, London, Canada.

Received: 17 October 2016 Accepted: 18 January 2017

Published online: 27 January 2017

\section{References}

1. Timmerman R, Paulus R, Galvin J, et al. Stereotactic body radiation therapy for inoperable early stage lung cancer. JAMA. 2010;303:1070-6.

2. Nguyen NP, Garland L, Welsh J, et al. Can stereotactic fractionated radiation therapy become the standard of care for early stage non-small cell lung carcinoma. Cancer Treat Rev. 2008:34:719-27.

3. Huang K, Dahele M, Senan S, et al. Radiographic changes after lung stereotactic ablative radiotherapy (SABR) - can we distinguish recurrence from fibrosis? A systematic review of the literature. Radiother Oncol. 2012; 102:335-42.

4. Tanvetyanon T, Clark JI, Campbell SC, et al. Neoadjuvant therapy: an emerging concept in oncology. South Med J. 2005;98:338-44.

5. Palma DA, Louie AV, Rodrigues GB. New strategies in stereotactic radiotherapy for oligometastases. Clin Cancer Res. 2015;21:5198-204.

6. Lagerwaard FJ, Haasbeek CJ, Smit EF, et al. Outcomes of risk-adapted fractionated stereotactic radiotherapy for stage I non-small-cell lung cancer. Int J Radiat Oncol Biol Phys. 2008;70:685-92.

7. Palma DA, Haasbeek CJ, Rodrigues GB, et al. Stereotactic ablative radiotherapy for comprehensive treatment of oligometastatic tumors (SABRCOMET): study protocol for a randomized phase II trial. BMC Cancer. 2012;12:305.

8. Schneider T, Reuss D, Warth A, et al. The efficacy of bipolar and multipolar radiofrequency ablation of lung neoplasms - results of an ablate and resect study. Eur J Cardiothorac Surg. 2011;39:968-73.

9. Chen F, Matsuo Y, Yoshizawa A, et al. Salvage lung resection for non-small cell lung cancer after stereotactic body radiotherapy in initially operable patients. J Thorac Oncol. 2010;5:1999-2002.
10. Hamamoto $Y$, Kataoka M, Yamashita M, et al. Lung-cancer related chest events detected by periodical follow-up CT after stereotactic body radiotherapy for stage I primary lung cancer: retrospective analysis of incidence of lung-cancer related chest events and outcomes of salvage treatment. Jpn J Radiol. 2012;30:671-5.

11. Neri S, Takahashi Y, Terashi T, et al. Surgical treatment of local recurrence after stereotactic body radiotherapy for primary and metastatic lung cancers. J Thorac Oncol. 2010;5:2003-7.

12. Allibhai Z, Cho BCJ, Taremi M, et al. Surgical salvage following stereotactic body radiotherapy for early-stage NSCLC. Eur Respir J. 2012;39:1039-42.

13. Chang JY, Senan S, Paul MA, et al. Stereotactic ablative radiotherapy versus lobectomy for operable stage I non-small-cell lung cancer: a pooled analysis of two randomised trials. Lancet Oncol. 2015;16:630-7.

14. Fernando HC, Landreneau RJ, Mandrekar SJ, et al. Thirty- and ninety-day outcomes after sublobar resection with and without brachytherapy for nonsmall cell lung cancer: results from a multicenter phase III study. J Thorac Cardiovasc Surg. 2011;142:1143-51.

15. Birkmeyer JD, Stukel TA, Siewers AE, et al. Surgeon volume and operative mortality in the United States. N Engl J Med. 2003;349:2117-27.

\section{Submit your next manuscript to BioMed Central and we will help you at every step:}

- We accept pre-submission inquiries

- Our selector tool helps you to find the most relevant journal

- We provide round the clock customer support

- Convenient online submission

- Thorough peer review

- Inclusion in PubMed and all major indexing services

- Maximum visibility for your research

Submit your manuscript at www.biomedcentral.com/submit
) Biomed Central 